\title{
Docosahexaenoic acid elevates trans-18:1 isomers but is not directly converted into trans-18:1 isomers in ruminal batch cultures
}

\author{
C. M. Klein and T. C. Jenkins ${ }^{1}$ \\ Department of Animal Science, Clemson University, Clemson, SC 29634-0311
}

\section{ABSTRACT}

Pathways of docosahexaenoic (DHA) biohydrogenation are not known; however, DHA is metabolized by ruminal microorganisms. The addition of DHA to the rumen alters the fatty acid profile of the rumen and milk and leads to increased trans-18:1 isomers, particularly trans-11 18:1. This study included 2 in vitro experiments to identify if the increase in trans-11 C18:1 was due to DHA being converted into trans-11 18:1 or if DHA stimulated trans-11 products from biohydrogenation of other fatty acids. In each experiment, ruminal microorganisms collected from a lactating Holstein cow were incubated in $10-\mathrm{mL}$ batch cultures for $0,6,24$, and $48 \mathrm{~h}$ and a uniformly ${ }^{13} \mathrm{C}$-labeled DHA was added to the cultures at $0 \mathrm{~h}$ as a metabolic tracer. Experiment 1 tested $0.5 \%$ DHA supplementation and experiment 2 examined 1,2, and 3\% DHA supplementation to determine if the level of DHA effected its conversion into trans-11 18:1. In both experiments, any fatty acid that was enriched with the ${ }^{13} \mathrm{C}$ label was determined to arise from DHA. Palmitic (C16:0), stearic (C18:0), all trans-18:1, eicosanoic (C20:0), and docosanoic (C22:0) acids were examined for enrichment. In experiment 1 , the amount of trans-18:1 isomers increased $0.415 \mathrm{mg}$ from 0 to $48 \mathrm{~h}$; however, no label was found in trans-18:1 at any time. Docosanoic acid was highly enriched at 24 $\mathrm{h}$ and $48 \mathrm{~h}$ to 20.2 and $16.3 \%$. Low levels of enrichment were found in palmitic and stearic acids. In experiment 2 , trans-18:1 isomers increased 185,256 , and $272 \%$ from 0 to $48 \mathrm{~h}$ when DHA was supplemented at 1,2, and $3 \%$, respectively; however, as in experiment 1, no enrichment occurred of any trans-18:1 isomer. In experiment 2 , low levels of label were found in palmitic and stearic acids. Enrichment of docosanoic acid decreased linearly with increased DHA supplementation. These studies showed that trans-18:1 fatty acids are not produced from DHA, supporting that DHA elevates trans-18:1 by modifying biohydrogenation pathways of other polyunsaturated fatty acids.

Received March 6, 2011.

Accepted May 25, 2011.

${ }^{1}$ Corresponding author: tjnkns@clemson.edu
Key words: docosahexaenoic acid (DHA), ruminal batch culture, trans fatty acid

\section{INTRODUCTION}

Fish oil contains a large amount of unsaturated, bioactive n-3 fatty acids including docosahexaenoic acid (DHA) and eicosapentaenoic acid (EPA). Supplementation of DHA has been associated with physiological benefits in many species. In dairy cows, DHA supplementation has been shown to alter the fatty acid composition of some parts of the reproductive system and $\mathrm{PGF}_{2 \alpha}$ secretions, which may, in turn, improve reproductive efficiencies (R. Mattos et al., 2004). To increase DHA intake, DHA can be included in dairy rations, either as a component of fish meal or as a fat supplement. When DHA is injected into the duodenum more DHA is transferred to milk than when DHA is injected into the rumen, indicating that DHA is hydrogenated in the rumen (Loor et al., 2004). Feeding DHA can modify the ruminal environment and decrease DMI, milk production, and milk fat production as well as alter fatty acid profiles (Donovan et al., 2000; Boeckaert et al., 2008).

It is widely known that ruminal microorganisms eliminate unsaturated 18-carbon fatty acids such as linoleic (C18:2) and linolenic (C18:3) acids through biohydrogenation; however, it is not well documented if the same processes occur for longer-chain fatty acids such as DHA (Jenkins et al., 2008). Supplementation of DHA results in increased levels of trans-18:1, particularly trans-11 18:1, in the rumen (Donovan et al., 2000; Cruz-Hernandez et al., 2007; Boeckaert et al., 2008). Because of the biological implications of trans-18:1 isomers, it is important to understand when and how they are produced. Trans-18:1 isomers are important intermediates of linoleic acid and linolenic acid biohydrogenation. Trans-10 18:1 is an indicator of milk fat depression in dairy cows, which is a concern in the dairy industry (Shingfield et al., 2009). Trans-11 18:1 can be converted to cis-9,trans-11 conjugated linoleic acid (CLA) by stearoyl-coenzyme A (CoA) desaturase in tissues (Pariza et al., 2001). Conjugated linoleic acid isomers, including CLA, are potent anticarcinogens (Pariza et al., 2001). 
The mechanism for the increase of trans-18:1 isomers in the rumen when DHA is supplemented has not been clearly documented. Possibly, DHA is converted into trans-18:1 isomers or DHA supplementation enhances trans-18:1 production from other 18-carbon unsaturated fatty acid biohydrogenation, or both. Our goal was to determine if DHA is converted into trans-18:1 isomers or if the increase in trans-18:1 is due to DHA altering other pathways. To determine this, we used a uniformly ${ }^{13} \mathrm{C}$-labeled DHA in ruminal batch cultures and looked for the presence of the ${ }^{13} \mathrm{C}$ label in trans-11 18:1 isomers. If label appeared in a fatty acid, it was regarded as an intermediate of DHA biohydrogenation.

\section{MATERIALS AND METHODS}

The rumen in vitro design was adapted from Sayre and Van Soest (1972) and AbuGhazaleh and Jenkins (2004b). For each set of batch cultures, a novel collection of rumen fluid was taken from 1 of 2 lactating, ruminally fistulated Holstein cows being fed 36.5 $\mathrm{kg} / \mathrm{d}$ of TMR $(56.5 \%$ corn silage, $3.7 \%$ alfalfa hay, $3.7 \%$ rye grass and oat haylage mix, and $36 \%$ grain mix by weight). Experiment 1 was completed with a single collection of rumen fluid, whereas experiment 2 included 3 separate collections of rumen fluid, with a novel collection for each replicate. In all cases, the rumen fluid was strained through 2 layers of cheesecloth, and then transported to the laboratory in a sealed and insulated container. At $0 \mathrm{~h}, 8 \mathrm{~mL}$ of media, $0.4 \mathrm{~mL}$ of reducing solution, and $2 \mathrm{~mL}$ of rumen fluid were added to screw-capped culture tubes $[15 \mathrm{~cm} \times 2 \mathrm{~cm}$ (experiment 1 ) or $15 \mathrm{~cm} \times 2.5 \mathrm{~cm}$ (experiment 2)] containing $100 \mathrm{mg}$ of substrate. Acids are produced by ruminal microorganisms and accumulate in batch cultures over time, which can interfere with microbial activities including decreased biohydrogenation (Martin and Jenkins, 2002). In this study, the media used was buffered with $1 \mathrm{~g}$ of ammonium bicarbonate/L and $8.75 \mathrm{~g}$ of sodium bicarbonate/L in an effort to maintain the $\mathrm{pH}$ and prevent any negative effects of low $\mathrm{pH}$, including preventing a decrease in trans-18:1 production AbuGhazaleh and Jenkins (2004b). The base substrate was composed of alfalfa pellets (50\%), ground corn (48\%), soybean meal $(22 \%)$, soybean hulls $(24 \%)$, dicalcium phosphate $(2.7 \%)$, trace mineral salt $(1.2 \%)$, and sodium bicarbonate $(1.6 \%)$, all ground through a $0.5-\mathrm{mm}$ screen. In both experiments, DHA treatments were added to the substrate in an injection of ethanol $(0.5$ $\mathrm{mg}$ of DHA in $15 \mu \mathrm{L}$ of ethanol in experiment 1 and 1 , 2 , or $3 \mathrm{mg}$ of DHA in $90 \mu \mathrm{L}$ of ethanol in experiment 2 ). For both experiments, DHA treatments were a uniformly ${ }^{13} \mathrm{C}$-labeled DHA methyl ester purchased from Cambridge Isotope Laboratories Inc. (Andover, MA) or a not-labeled DHA purchased from $\mathrm{Nu}$-Chek Prep Inc. (Elysian, MN). The 0-h cultures were acidified with $6 \mathrm{~N} \mathrm{HCl}$ immediately before the addition of ruminal microorganisms, and then stored at $-15^{\circ} \mathrm{C}$ to prevent biohydrogenation. The remaining cultures were flushed with $\mathrm{CO}_{2}$, capped, and incubated at $39^{\circ} \mathrm{C}$. At 6-, 24-, and 48-h sampling times, the appropriate cultures were acidified with $6 \mathrm{~N} \mathrm{HCl}$ and stored at $-15^{\circ} \mathrm{C}$.

\section{Chemical Analysis}

Whole cultures were analyzed for VFA or long-chain fatty acids (LCFA). Cultures designated for VFA analysis were thawed and centrifuged at 15,780 $\times \mathrm{g}$, and then $1 \mathrm{~mL}$ of supernatant was collected and 100 $\mu \mathrm{L}$ of 2-ethylbutyric acid $(86 \mu \mathrm{mol} / 100 \mathrm{~mL})$ was added as an internal standard. The samples were analyzed by GC on a Supelco $30 \mathrm{~m} \times 0.25 \mathrm{~mm} \times 0.25 \mu \mathrm{m}$ fused silica capillary column (Supelco Inc., Bellefonte, PA; Fellner et al., 1997).

Cultures designated for LCFA analysis were lyophilized, then fatty acids were converted to fatty acid methyl esters (FAME) using a 2-step methylation procedure (Jenkins, 2010). Methyl esters were separated by $\mathrm{GC}$ on a $100 \mathrm{~m} \times 0.25 \mathrm{~mm} \times 0.2 \mathrm{um}$ column $(\mathrm{Su}-$ pelco SP-2560; Supelco Inc.) and detected by a flame ionization detector. Individual FAME were quantified by comparison to a C17:0 or C19:0 internal standard.

Enrichment of ${ }^{13} \mathrm{C}$ labeling was measured by GC-MS using chemical ionization with methane as the reagent gas. The FAME samples were separated on a $100 \mathrm{~m} \times$ $0.25 \mathrm{~mm} \times 0.2$ um column (Varian CP7489; Agilent Technologies, Santa Clara, CA). The mass selective detector was set to scan mode, scanning masses between 200 and 420 with a threshold count level of 300 . The amount of ${ }^{13} \mathrm{C}$ in each fatty acid was reported as enrichment, the ratio of ${ }^{13} \mathrm{C}$ to total carbon in the fatty acid. Enrichment was calculated as $\{(\mathrm{M}+\mathrm{n}) /[\mathrm{M}+(\mathrm{M}+$ $\mathrm{n})] \times 100\}_{\text {Labeled }}-\{(\mathrm{M}+\mathrm{n}) /[\mathrm{M}+(\mathrm{M}+\mathrm{n})] \times 100\}_{\text {Not }}$ labeled, where $\mathrm{M}$ is the molecular ion and $\mathrm{n}$ is the number of carbons in the fatty acid (Mosley et al., 2002). The molecular ion represents the naturally abundant ${ }^{12} \mathrm{C}$ and $\mathrm{M}+\mathrm{n}$ represents the ${ }^{13} \mathrm{C}$ label. The enrichment of not labeled cultures was subtracted from the labeled cultures to account for any background levels of ${ }^{13} \mathrm{C}$.

\section{Experiment 1}

In experiment 1 , treatment groups were $0.5 \%{ }^{13} \mathrm{C}$ labeled DHA (L), 0.5\% not-labeled DHA (NL), and nonfat-added control (CTL). Duplicate cultures were maintained for each treatment at each time and analysis. Half of the cultures were analyzed for VFA and the other half for LCFA. 


\section{Experiment 2}

In experiment 2, we examined the effects of increasing levels of DHA in batch cultures. The treatment groups were $1 \%{ }^{13} \mathrm{C}$-labeled DHA (1L), $2 \%{ }^{13} \mathrm{C}$-labeled DHA $(\mathbf{2 L}), 3 \%{ }^{13} \mathrm{C}$-labeled DHA $(\mathbf{3 L}), 1 \%$ not-labeled DHA (1NL), 2\% not-labeled DHA (2NL), 3\% not-labeled DHA (3NL), and CTL. All cultures were analyzed for LCFA. This experiment was replicated 3 times in a randomized complete block design, with blocks being each in vitro run that started with a fresh collection of rumen inocula.

\section{Statistical Methods}

All statistical analysis was completed using SAS version 9.2 (SAS Institute Inc., Cary, NC). Fatty acids were determined to arise from DHA if enrichments differed from zero by $t$-test $(P<0.01)$. A least squares means ANOVA in the GLM procedure using incubation time and DHA level as sources of variation was used to test time and treatment effects. Experiment 1 was a completely randomized design with sources of variation being treatment and time, and experiment 2 was a randomized complete block design with sources of variation being treatment, time, and replicate (i.e., novel collection of rumen fluid inocula). In experiment 2, orthogonal contrast statements were used to determine if linear or quadratic effects of DHA supplementation level existed. Throughout, $P \leq 0.05$ was considered to be significant unless otherwise noted.

\section{RESULTS AND DISCUSSION}

\section{Experiment 1}

Ruminal batch cultures are used as a model of the rumen environment and, thus, should reasonably duplicate in vivo fermentation conditions. In experiment 1 , an accumulation of VFA occurred from an average of 23 $\mu \mathrm{mol} / \mathrm{mL}$ at $0 \mathrm{~h}$ to $88 \mu \mathrm{mol} / \mathrm{ml}$ at $48 \mathrm{~h}$ (Figure 1). The increase in VFA in the batch cultures indicates that fermentation was occurring and that the microorganisms were viable. No effect of either the labeled or notlabeled DHA was found on either the concentrations or proportions of VFA, indicating that the cultures were viable with and without the DHA treatments. Figure $2 \mathrm{~A}$ shows that unsaturated fatty acids, including linoleic acid, linolenic acid, and DHA decreased over time in experiment 1 . This was accompanied by an increase in saturated fatty acids, including 18:0 and 22:0 (Figure $2 \mathrm{~A}$ ), and monounsaturated fatty acids (Figure $2 \mathrm{~B}$ ). The shift from unsaturated to saturated fatty acids indicates that biohydrogenation was occurring in the

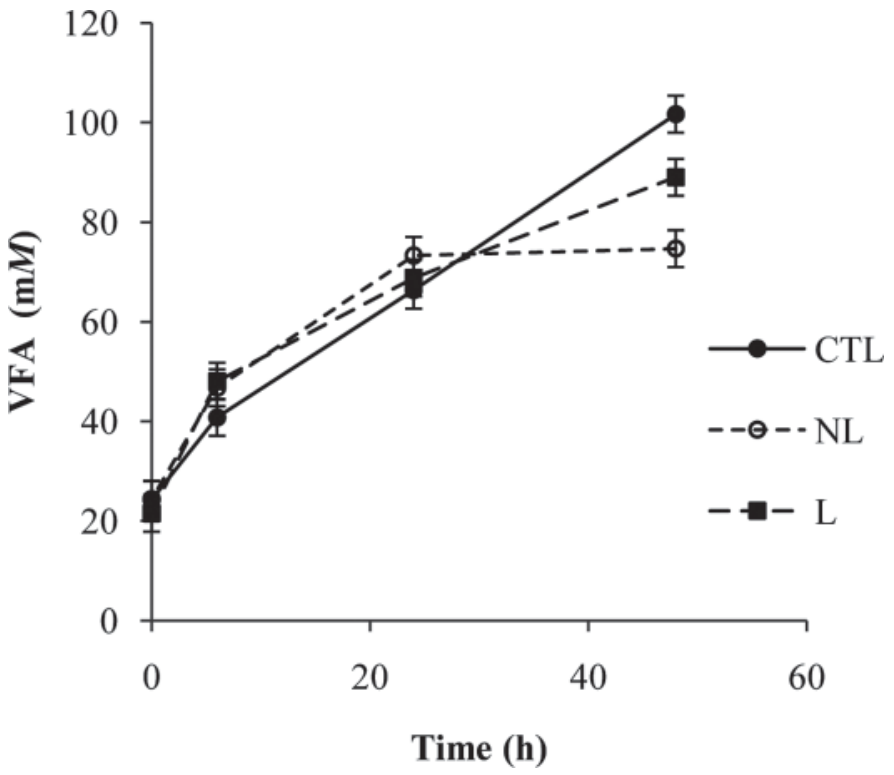

Figure 1. The accumulation of total VFA in experiment 1 cultures over time when either no fat (control; CTL), $0.5 \%$ not-labeled docosahexaenoic acid (DHA; NL), or $0.5 \%{ }^{13} \mathrm{C}$-labeled DHA (L) was added to cultures of mixed ruminal microorganisms. Each point is the mean of 2 replicates and pooled standard errors are shown.

batch cultures. Combined, the VFA accumulation and biohydrogenation signify that the microorganisms were active and responded similar to in vivo response.

Previous studies have shown that adding DHA to the rumen alters the fatty acid profile in the rumen and in milk, including increased trans-18:1 isomers (AbuGhazaleh and Jenkins, 2004b; Boeckaert et al., 2008). As expected, accumulation of trans-18:1 fatty acids occurred in the cultures in experiment 1, particularly trans-11 18:1 (Figure 2B). The disappearance of DHA and accumulation of trans-18:1 isomers indicate that these cultures are good models for a ruminant being fed DHA and can be used to show if DHA is converted into trans-18:1 isomers.

Although an increase in trans-18 isomers occurred in the L and NL cultures, no enrichment occurred of any trans-18:1 isomer at any time (Figure 3 ). This indicates that DHA was not a direct contributor to the increase in trans-18:1 isomers in experiment 1. Additionally, no enrichment of oleic (18:1) or eicosanoic (20:0) acids occurred. These fatty acids are not known to be produced from DHA and the absence of label is an indicator that the label was not indiscriminately appearing in fatty acids that are not produced from DHA. Figure 3 shows the fatty acid that was found to be substantially enriched was docosanoic acid (C22:0), which was $16.3 \%$ labeled at $48 \mathrm{~h}$. Known pathways of 18-carbon linoleic and linolenic acid biohydrogenation terminate in saturated stearic acid (Lock and Bauman, 2004). The 
A

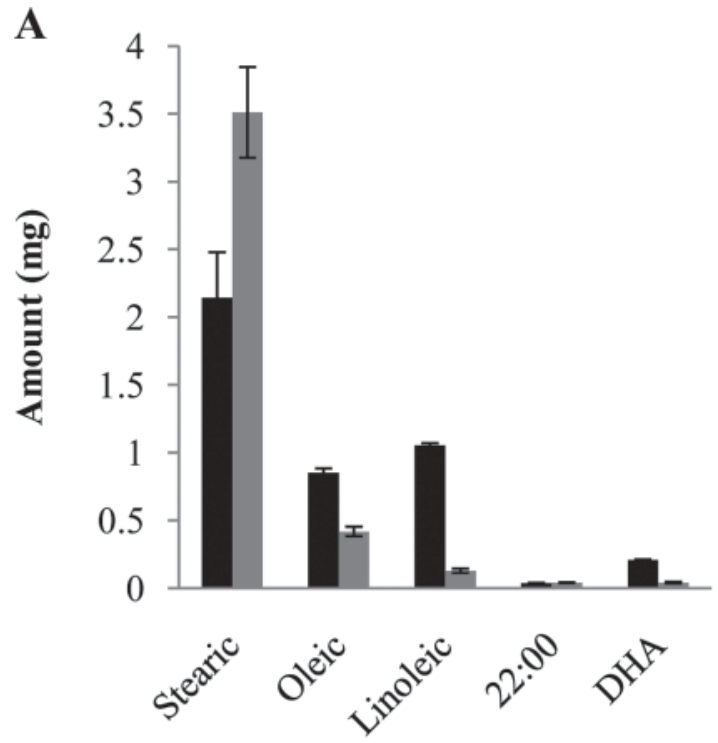

Fatty acid

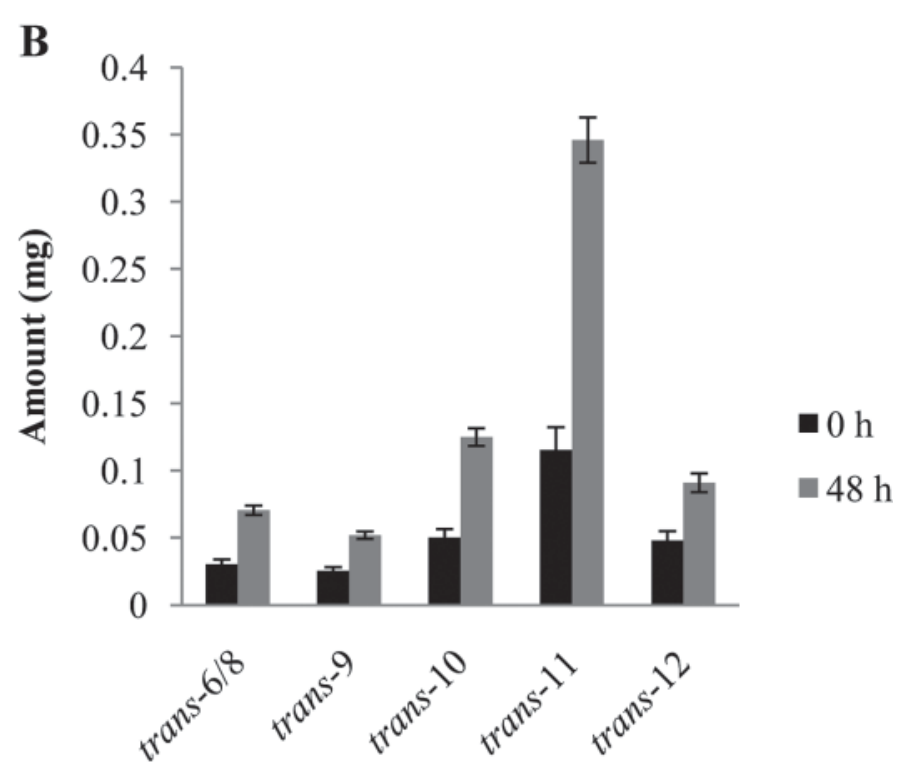

C18:1 fatty acid

Figure 2. (A) The experiment 1 changes in amount of stearic, oleic, linoleic, docosanoic, and docosahexaenoic fatty acids (DHA) at 0 and $48 \mathrm{~h}$ when $0.5 \%{ }^{13} \mathrm{C}$-labeled DHA was added to cultures of mixed ruminal microorganisms. Each bar is the mean of 2 replicates and pooled standard errors are shown. (B) The changes in amount of trans-6/8, trans-9, trans-10, trans-11, and trans-12 C18:1 fatty acids at 0 and $48 \mathrm{~h}$ when $0.5 \%$ labeled DHA was added to cultures of mixed ruminal microorganisms. Each bar is the mean of 2 replicates and pooled standard errors are shown.

appearance of label in docosanoic acid may indicate that similar pathways of isomerization and saturation occur in 22 carbon fatty acids, resulting in docosanoic acid. In addition to the label in docosanoic acid, less

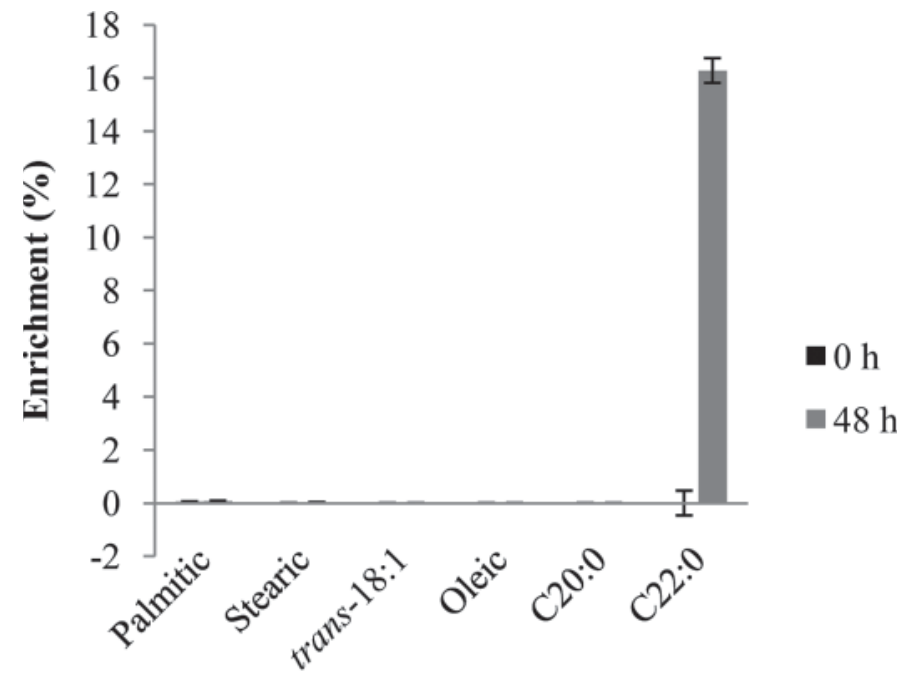

Fatty acid

Figure 3. The percentages of ${ }^{13} \mathrm{C}$ enrichment of palmitic, stearic, trans-18:1, oleic, eicosanoic, and docosanoic acids at 0 and $48 \mathrm{~h}$ in experiment 1 when $0.5 \%{ }^{13} \mathrm{C}$-labeled docosahexaenoic acid (DHA) was added to cultures of mixed ruminal microorganisms. Enrichments were detected in palmitic, stearic, and docosanoic acids $(P$-value $<0.05)$. Each bar is the mean of 2 replicates and pooled standard errors are indicated.

than $0.1 \%$ enrichment occurred in palmitic and stearic acids (Figure 3). Experiment 1 results indicate that $0.5 \%$ DHA supplementation results in an increase in the amount of trans-18:1, but not conversion of DHA into trans-18:1 isomers. Labeled DHA was saturated to docosanoic acid in experiment 1 , which may indicate that DHA biohydrogenation parallels known biohydrogenation pathways of linoleic and linolenic acids where double bonds are isomerized, and then eliminated, to produce a saturated fatty acid of the same chain length as the initial unsaturated fatty acid.

\section{Experiment 2}

In experiment 2, 3 levels of DHA supplementation were tested to determine if the level of supplementation altered the intermediates produced from DHA, including the formation of trans-18:1 isomers. Table 1 shows the major changes in fatty acid contents for CTL and 1,2 , and $3 \%$ DHA at 0 and $48 \mathrm{~h}$ in experiment 2 . In all cultures, accumulations of saturated fatty acids and decreasing levels of unsaturated fatty acids occurred in the cultures over time (Table 1). The shift in fatty acid profile indicates that the batch cultures were viable and the biohydrogenation of fatty acids was comparable to what would occur in the rumen (Jenkins et al. 2008). In this experiment, DHA disappeared from cultures at each level of supplementation, so DHA products ap- 
peared labeled in these cultures. No increase occurred in trans-10 18:1 in this study, which is consistent with the findings of Vlaeminck et al. (2008), but differs from the findings of Or-Rashid et al. (2008). The variation in trans-10 18:1 could be due to feeding pure DHA rather than fish oil, the effect of other confounding dietary components, or a time lag in the effect of DHA on trans-10 18:1. Vlaeminck et al. (2008) saw differences in trans-10 18:1 in rumen fluid that was adapted to marine algae for $21 \mathrm{~d}$ but not when pure DHA was added to cultures for $24 \mathrm{~h}$. This supports either a lag in effect on trans-10 18:1 or confounding effects of other marine algae components. The changes in the fatty acid profile are typical of ruminal contents and support that these cultures were an adequate model of rumen activity and were capable of determining products of DHA in the rumen.

Trans-11 18:1 accumulated in all cultures over time with higher accumulations associated with higher levels of DHA supplementation (Figure 4). AbuGhazaleh and Jenkins (2004b) previously reported a correlation between DHA supplementation and trans-11 18:1; however, were not able to identify the source of the trans-11 18:1. If DHA is a source of trans-11 18:1 then enrichment of trans-11 18:1 should increase with level of DHA in this study.

Although a positive linear correlation existed between trans-11 18:1 accumulation and increased DHA supplementation, no enrichment of trans-11 18:1 occurred at any time or level of DHA supplementation (Figure 5). Additionally, as seen in experiment 1 , no enrichment of $t 6, t 8, t 9, t 10$, or $t 12$ 18:1 isomers occurred. The batch cultures were maintained for $48 \mathrm{~h}$, which exceeds the amount of time that a fatty acid would remain in the rumen and ensures that the lack of conversion of DHA into trans-18:1 was not due to time constraints. Whenever a 0 result is recorded, it is important to understand the sensitivity of the techniques and instruments used and determine if they are sufficient to make conclusions about the biology or if they indicate insufficient measurement techniques. In experiment 2 , an average of $0.531 \mathrm{mg}$ of trans-11 was found per culture, which was equivalent to an average of $2377669 \mathrm{M}$ and $\mathrm{M}+18$ isotope counts by the mass selective detector. The detector was programmed to record all counts above 300 , which would be theoretically equivalent to $0.0126 \%$ enrichment or $0.067 \mu \mathrm{g}$ of labeled trans -11 18:1 in the average trans-11 18:1 peak. Based on this level of sensitivity, it seems reasonable to conclude that, as no label was detected, DHA was not converted into trans-11 18:1 in this experiment. This confirms the results of experiment 1 , that DHA causes an increase in trans-11 accumulation in ruminal batch cultures, but it is not a direct contributor to the increase. Instead, DHA is altering the rumen processes, perhaps by altering the microorganisms that are present, or altering the reaction pathways, or both, ultimately resulting in an increase in trans-11 18:1.

Table 1. The changes in amount of fatty acid (mg/culture) when no fat was added (control; CTL) and when 1,2 , or $3 \%$ docosahexaenoic acid (DHA) was added to cultures of ruminal microorganisms in experiment $2^{1}$

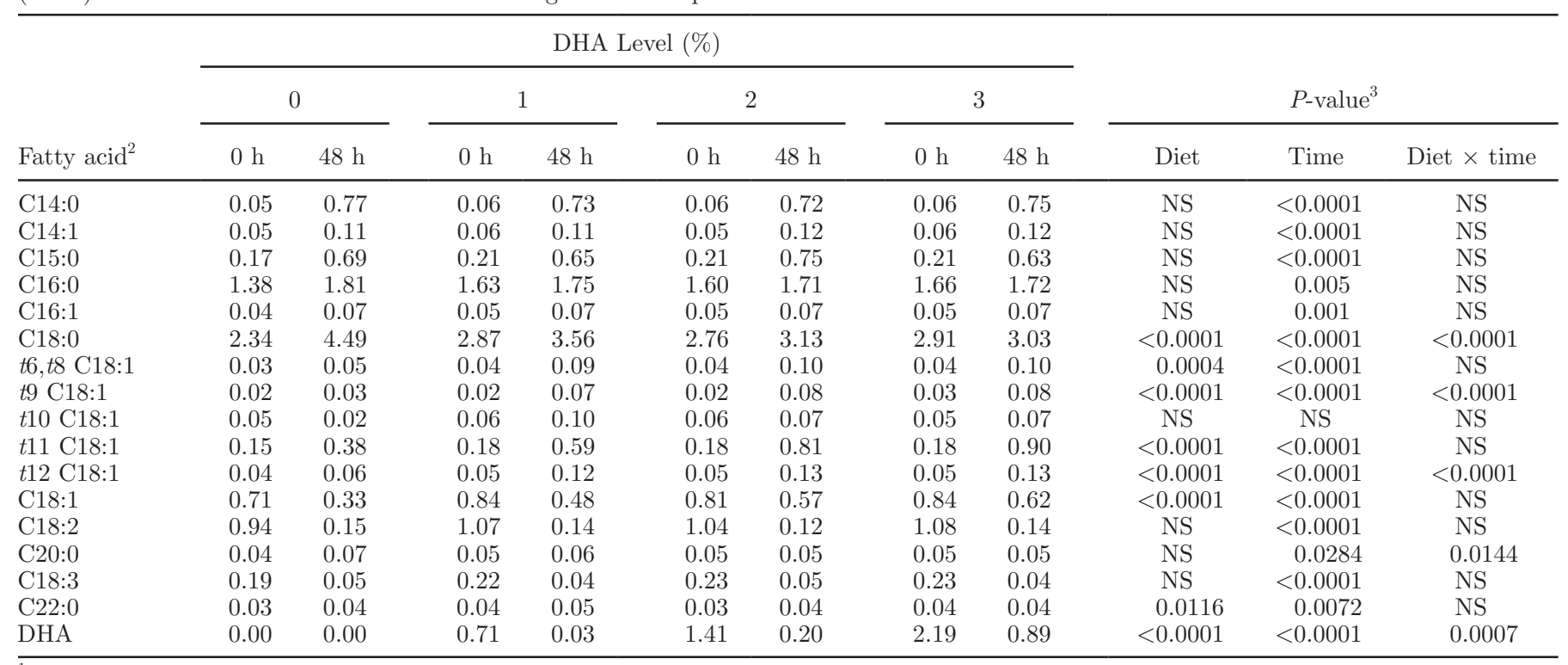

${ }^{1}$ CTL values are means of 3 replicates and DHA values are means of 3 replicates where not-labeled DHA was added and 3 replicates where ${ }^{13} \mathrm{C}$-labeled DHA was added to the cultures.

${ }^{2} t=$ trans

${ }^{3} P$-values indicate an effect of diet, time, or an interaction between diet and time $(P$-value $<0.05)$. 


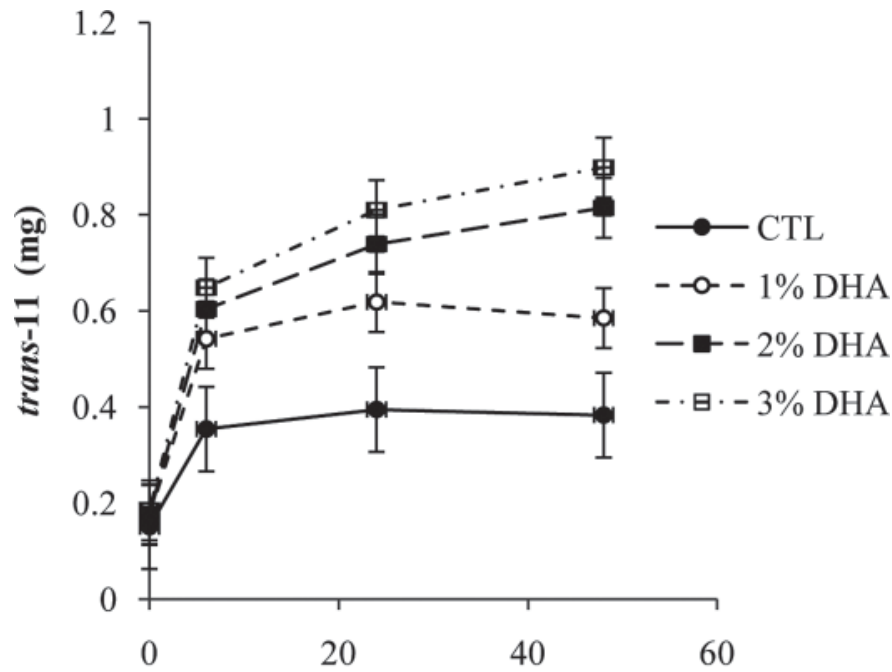

Time (h)

Figure 4. The amount of trans-11 18:1 fatty acid that accumulated in ruminal batch cultures over time in experiment 2 when no fat (control; CTL), 1\% docosahexaenoic acid (DHA), $2 \%$ DHA, or $3 \%$ DHA was added. The CTL data points are means of 3 replicates and DHA data points are means of 3 replicates where not-labeled DHA was added and 3 replicates where ${ }^{13} \mathrm{C}$-labeled DHA was added. Pooled standard errors are shown.

In addition to trans-18:1 fatty acids, oleic, linoleic, linolenic, and eicosanoic acids were examined and were not enriched at any time or level of DHA (data not shown). As in experiment 1, palmitic and stearic acids were enriched less than $0.1 \%$ (Figure 6 ). Although the enrichment of stearic acid was statistically significant,

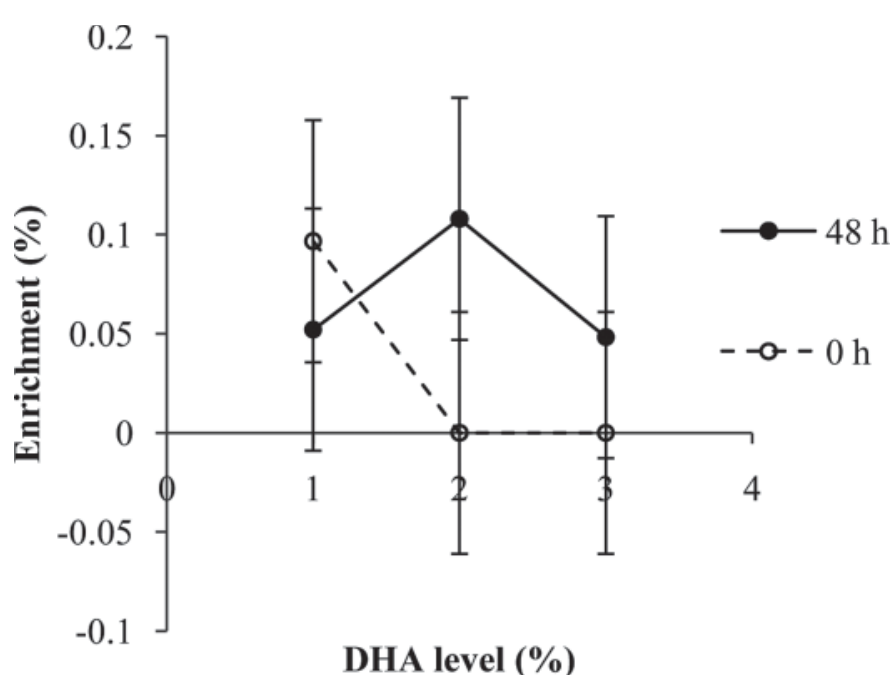

Figure 5. Experiment 2 enrichments of trans-11 $18: 1$ at 0 and 48 $\mathrm{h}$ by level of docosahexaenoic acid (DHA) supplementation in experiment 2. No significant enrichment occurred at any time $(P$-value $<$ $0.05)$. Each data point is the mean of 3 replicates and pooled standard errors are indicated. the biological implications are limited. The highest enrichments were found at $48 \mathrm{~h}$, when $3.03 \mathrm{mg}$ of stearic acid was in the $3 \%$ DHA cultures and stearic acid was $0.09 \%$ enriched. In these cultures a potential existed for stearic acid to become $43 \%$ enriched at $48 \mathrm{~h}$ if all of the DHA that disappeared had been converted to stearic acid. Multiplying the amount of stearic acid by the enrichment, $<0.003 \mathrm{mg}$ of the stearic acid was calculated to be derived from DHA, which would be equivalent to $<0.10 \%$ of DHA being converted to stearic acid. The authors are not aware of any known pathways where ruminal microorganisms shorten the carbon chain length of fatty acids and do not believe that the slight levels of enrichment of stearic and palmitic acids found in these experiments are substantial enough to conclude that these pathways exist.

Docosanoic acid was $12.7 \%$ labeled at $48 \mathrm{~h}$ in the $1 \%$ DHA-supplemented cultures; however, a linear decrease in docosanoic acid enrichment occurred as DHA supplementation level increased (Figure 6). At high levels, Kim et al. (2000) found that linoleic acid inhibits its own biohydrogenation, and AbuGhazaleh and Jenkins (2004a) noted decreased percent disappearance of DHA and eicosapentaenoic acid with increased supplementation. Although DHA was lost at each supplementation level in this experiment, the amount of DHA that disappeared by $48 \mathrm{~h}$ decreased from $95 \%$ at $1 \%$ DHA to $63 \%$ at $3 \%$ DHA (Figure 7 ). The decrease in docosanoic acid enrichment, combined with the decreased percent loss of DHA, indicates that DHA biohydrogenation is inhibited when DHA levels are high.

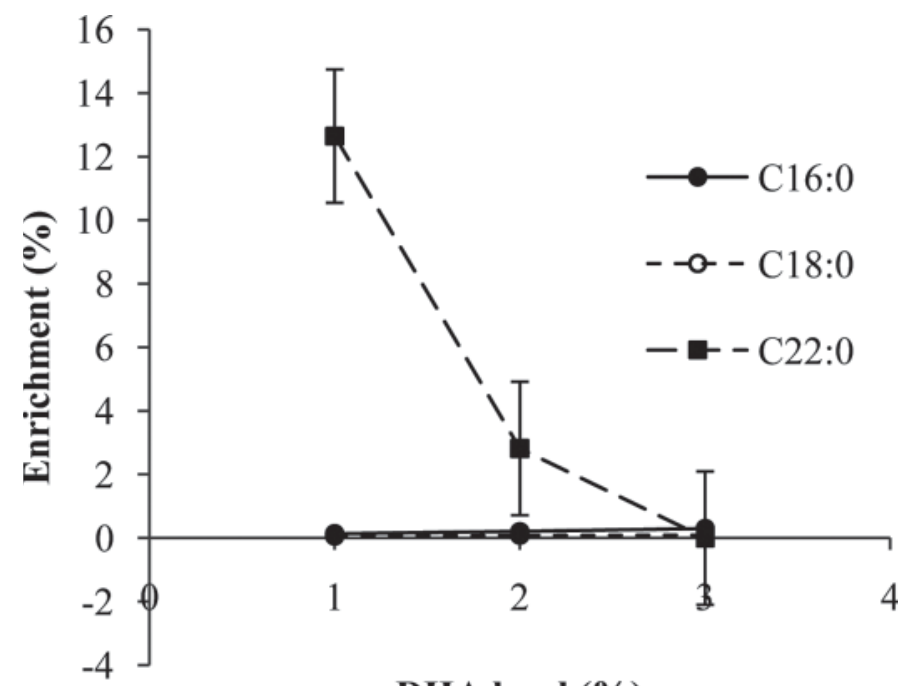

DHA level (\%)

Figure 6. Experiment 2 enrichments of palmitic (C16:0), stearic (C18:0), and docosanoic acids (C22:0) at $48 \mathrm{~h}$ as level of docosahexaenoic acid (DHA) increased from 1 to $3 \%$. Each point is the mean of 3 replicates and pooled standard errors are shown. 


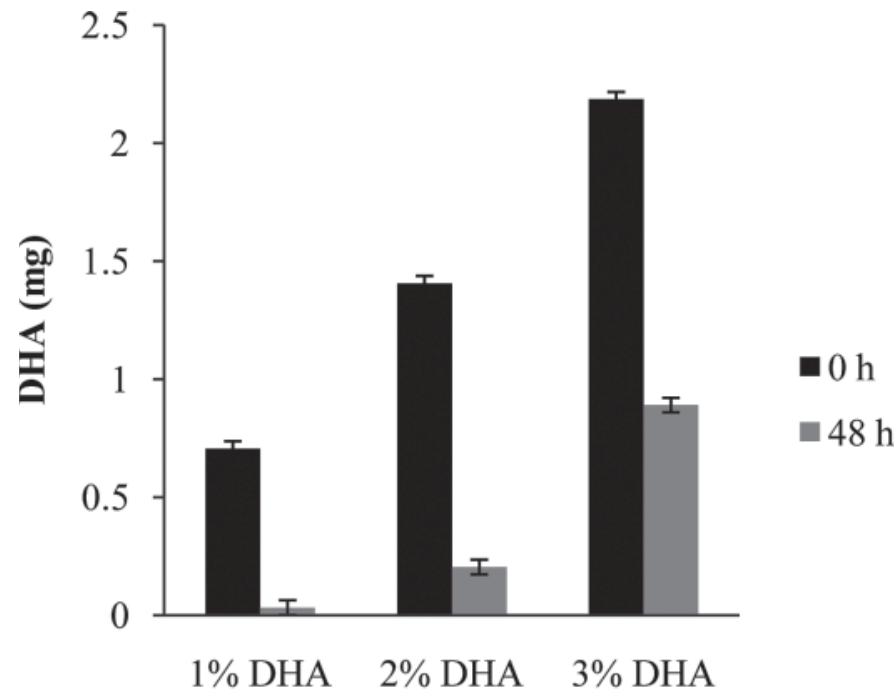

Diet

Figure 7. The amount of docosahexaenoic acid (DHA) that was present in experiment 2 cultures at 0 and 48 hy level of DHA supplementation. Each bar is the mean of 3 replicates where not-labeled DHA was added and 3 replicates where ${ }^{13} \mathrm{C}$-labeled DHA was added. Pooled standard errors are shown.

These studies examined several levels of DHA supplementation at extended time points and conclusively determined that under these conditions, ruminal bacteria do not convert DHA to trans-18:1 isomers. The batch culture design is ideal to measure the culture contents precisely, ensuring true measures of inputs and products; however, some constraints exist. The cultures accumulate products, which could contribute to feedback inhibition. The cultures are highly buffered to enable the microorganisms to grow for extended periods of time without waste product removal. These cultures only looked at the effects of time and level of DHA and did not examine confounding effects of other fat or carbohydrate sources. As such, these cultures are conclusive that under these conditions, the microorganisms do not convert DHA to trans-18:1, but cannot conclude that under other circumstances ruminal microorganisms are not capable of this conversion.

Although differences exist between batch cultures and the rumen, several inferences can be drawn from this study about the effects of DHA on a ruminant animal. Although DHA is not converted into trans-18:1 isomers, it does result in an increase in these isomers. An increase in trans-11 18:1 will lead to increases in cis-9 trans-11 CLA in tissues. This study found no conversion of DHA into trans-10 18:1, which may indicate that DHA directly does not cause milk fat depression, but instead, other components of fish oil or interactions between DHA and other feed components are respon- sible for the decrease in milk fat production, such as those reported by Whitlock et al. (2002). Understanding the mechanisms and pathways involved in ruminal biohydrogenation of fatty acids is essential to developing and implementing feeding strategies that produce the desired fatty acid composition and beneficially affect milk production. That DHA is not directly converted into trans-11 18:1 and, instead, is otherwise affecting its synthesis is an important step in elucidating the pathways of trans-11 18:1 formation and the pathways of DHA biohydrogenation.

\section{CONCLUSIONS}

Although DHA supplementation results in increased levels of trans-18:1 fatty acids, DHA is not directly converted into trans-18:1 isomers. Docosanoic acid is produced directly from DHA. As DHA is converted into docosanoic acid, it may be hydrogenated in a manner that resembles known biohydrogenation pathways of linoleic and linolenic acids. If this is the case, a myriad of 22-carbon unsaturated fatty acids with unknown biological activity likely are produced from DHA in the rumen.

\section{ACKNOWLEDGMENTS}

This project was supported by National Research Initiative Competitive Grant no. 2008-35206-18835 from the USDA National Institute of Food and Agriculture (Washington, DC). Technical Contribution No. 5933 of the Clemson University Experiment Station (Clemson, $\mathrm{SC})$.

\section{REFERENCES}

AbuGhazaleh, A. A., and T. C. Jenkins. 2004a. Disappearance of docosahexaenoic and eicosapentaenoic acids from cultures of mixed ruminal microorganisms. J. Dairy Sci. 87:645-651.

AbuGhazaleh, A. A., and T. C. Jenkins. 2004b. Short communication: Docosahexaenoic acid promotes vaccenic acid accumulation in mixed ruminal cultures when incubated with linoleic acid. J. Dairy Sci. 87:1047-1050.

Boeckaert, C., B. Vlaeminck, J. Dijkstra, A. Issa-Zacaria, T. Van Nespen, W. Van Straalen, and V. Fievez. 2008. Effect of dietary starch or micro algae supplementation on rumen fermentation and milk fatty acid composition of dairy cows. J. Dairy Sci. 91:47144727.

Cruz-Hernandez, C., J. K. G. Kramer, J. J. Kennelly, D. R. Glimm, B. M. Sorensen, E. K. Okine, L. A. Goonewardene, and R. J. Weselake. 2007. Evaluating the conjugated linoleic acid and trans 18:1 isomers in milk fat of dairy cows fed increasing amounts of sunflower oil and a constant level of fish oil. J. Dairy Sci. 90:3786-3801.

Donovan, D. C., D. J. Schingoethe, R. J. Baer, J. Ryali, A. R. Hippen, and S. T. Franklin. 2000. Influence of dietary fish oil on conjugated linoleic acid and other fatty acids in milk from lactating dairy cows. J. Dairy Sci. 83:2620-2628.

Fellner, V., F. D. Sauer, and J. K. G. Kramer. 1997. Effect of nigericin, monensin, and tetronasin on biohydrogenation in continuous flowthrough ruminal fermenters. J. Dairy Sci. 80:921-928. 
Jenkins, T. C. 2010. Technical note: Common analytical errors yielding inaccurate results during analysis of fatty acids in feed and digesta samples. J. Dairy Sci. 93:1170-1174.

Jenkins, T. C., R. J. Wallace, P. J. Moate, and E. E. Mosley. 2008. Board-invited review: Recent advances in biohydrogenation of unsaturated fatty acids within the rumen microbial ecosystem. J. Anim. Sci. 86:397-412.

Kim, Y. J., R. H. Liu, D. R. Bond, and J. B. Russell. 2000. Effect of linoleic acid concentration on conjugated linoleic acid production by Butyrivibrio fibrisolvens A38. Appl. Environ. Microbiol. 66:5226-5230

Lock, A. L., and D. E. Bauman. 2004. Modifying milk fat composition of dairy cows to enhance fatty acids beneficial to human health. Lipids 39:1197-1206.

Loor, J. J., M. Doreau, J. M. Chardigny, A. Ollier, J. L. Sebedio, and Y. Chilliard. 2004. Effects of ruminal or duodenal supply of fish oil on milk fat secretion and profiles of trans-fatty acids and conjugated linoleic acid isomers in dairy cows fed maize silage. Anim. Feed Sci. Technol. 119:227-246.

Martin, S. A., and T. C. Jenkins. 2002. Factors affecting conjugated linoleic acid and trans-C18:1 fatty acid production by mixed ruminal bacteria. J. Anim. Sci. 80:3347-3352.

Mattos, R., C. R. Staples, A. Arteche, M. C. Wiltbank, F. J. Diaz, T. C. Jenkins, and W. W. Thatcher. 2004. The effects of feeding fish oil on uterine secretion of $\mathrm{PGF}_{2 \alpha}$, milk composition, and metabolic status of periparturient Holstein cows. J. Dairy Sci. 87:921-932.
Mosley, E. E., G. L. Powell, M. B. Riley, and T. C. Jenkins. 2002. Microbial biohydrogenation of oleic acid to trans isomers in vitro. J. Lipid Res. 43:290-296.

Or-Rashid, M. M., J. K. G. Kramer, M. A. Wood, and B. W. McBride. 2008. Supplemental algal meal alters the ruminal trans-18:1 fatty acid and conjugated linoleic acid composition in cattle. J. Anim. Sci. 86:187-196.

Pariza, M. W., Y. Park, and M. E. Cook. 2001. The biologically active isomers of conjugated linoleic acid. Prog. Lipid Res. 40:283-298.

Sayre, K. D., and P. J. Van Soest. 1972. Comparison of types of fermentation vessels for an in vitro artificial rumen procedure. J. Dairy Sci. 55:1496-1498.

Shingfield, K. J., A. Saebø, P. C. Saebø, V. Toivonen, and J. M. Griinari. 2009. Effect of abomasal infusions of a mixture of octadecenoic acids on milk fat synthesis in lactating cows. J. Dairy Sci. 92:4317-4329.

Vlaeminck, B., G. Mengistu, V. Fievez, L. de Jonge, and J. Dijkstra. 2008. Effect of in vitro docosahexaenoic acid supplementation to marine algae-adapted and unadapted rumen inoculum on the biohydrogenation of unsaturated fatty acids in freeze-dried grass. J. Dairy Sci. 91:1122-1132.

Whitlock, L. A., D. J. Schingoethe, A. R. Hippen, K. F. Kalscheur, R. J. Baer, N. Ramaswamy, and K. M. Kasperson. 2002. Fish oil and extruded soybeans fed in combination increase conjugated linoleic acids in milk of dairy cows more than when fed separately. J. Dairy Sci. 85:234-243. 